\title{
Hsp-27 levels and thrombus burden relate to clinical outcomes in patients with ST-segment elevation myocardial infarction
}

\author{
Maozhou Tiann ${ }^{1, *}$, Lingmin Zhu ${ }^{2, *}$, Hongyang Lin ${ }^{3, *}$, Qiaoyan Lin ${ }^{2, *}$, Peng Huang ${ }^{2, *}$, \\ Xiao $\mathrm{Yu}^{4}$ and Yanyan Jing ${ }^{2}$ \\ ${ }^{1}$ Department of Cardiac Surgery, The Affiliated Yantai Yuhuangding Hospital of Qingdao University, Yantai, Shangdong \\ 264000, P.R. China \\ ${ }^{2}$ Department of Cardiology, The Affiliated Yantai Yuhuangding Hospital of Qingdao University, Yantai, Shangdong 264000, \\ P.R. China \\ ${ }^{3}$ Emergency Centre, The Affiliated Yantai Yuhuangding Hospital of Qingdao University, Yantai, Shangdong 264000, P.R. China \\ ${ }^{4}$ Department of Endocrinology, The Affiliated Yantai Yuhuangding Hospital of Qingdao University, Yantai, Shangdong 264000, \\ P.R. China \\ ${ }^{*}$ These authors are contributed equally to this work
}

Correspondence to: Xiao Yu, email: yuxiaoshandong@sina.com

Yanyan Jing, email: jingyanyansd@sina.com

Keywords: Hsp-27, thrombus burden, STEMI, major adverse cardiovascular event

Received: December 06, 2016

Accepted: April 26, 2017

Published: May 13, 2017

Copyright: Tian et al. This is an open-access article distributed under the terms of the Creative Commons Attribution License 3.0 (CC BY

3.0), which permits unrestricted use, distribution, and reproduction in any medium, provided the original author and source are credited.

\section{ABSTRACT}

High thrombus burden, subsequent distal embolization, and myocardial noreflow remain a large obstacle that may negate the benefits of urgent coronary revascularization in patients with ST-segment elevation myocardial infarction (STEMI). However, the biological function and clinical association of Hsp-27 with thrombus burden and clinical outcomes in patients with STEMI is not clear. Consecutive patients $(n=146)$ having STEMI undergoing primary percutaneous coronary intervention (PPCI) within 12 hours from the onset of symptoms were enrolled in this prospective study in the Affiliated Yantai Yuhuangding Hospital of Qingdao University, Yantai, Shangdong, P.R. China. Patients were divided into low thrombus burden and high thrombus burden groups. The present study demonstrated that patients with highthrombus burden had higher plasma Hsp-27 levels ([32.0 \pm 8.6 vs. $58.0 \pm 12.3] \mathrm{ng} / \mathrm{mL}$, $P<0.001)$. The median value of Hsp-27 levels in all patients with STEMI was $45 \mathrm{ng} / \mathrm{mL}$. Using the receiver operating characteristic (ROC) curve analysis, plasma Hsp-27 levels were of significant diagnostic value for high thrombus burden (AUC, $0.847 ; 95 \%$ CI, 0.775-0.918; $P<0.01$ ). The multivariate cox regression analysis demonstrated that Hsp-27 > $45 \mathrm{ng} / \mathrm{mL}$ (HR 2.801, 95\% CI 1.296-4.789, $P=0.001$ ), were positively correlated with the incidence of major adverse cardiovascular events (MACE). KaplanMeier survival analysis demonstrated that MACE-free survival at 180-day follow-up was significantly lower in patients with Hsp-27 $>45 \mathrm{ng} / \mathrm{mL}(\log \operatorname{rank}=10.28, P<0.001)$.

Our data demonstrate that plasma Hsp-27 was positively correlated with high thrombus burden and the incidence of MACE in patients with STEMI who underwent PPCI.

\section{INTRODUCTION}

Intracoronary thrombus formation due to the rupture of an atherosclerotic plaque and subsequently reducing coronary blood flow constitute the main pathophysiology underlying ST-segment elevation myocardial infarction (STEMI) [1]. Primary percutaneous coronary intervention
(pPCI) of the infarct-related artery in patients with STEMI should be referred for thrombolytic therapy as soon as possible [1]. However, high thrombus burden may increase infarct size by blocking microvascular areas and causing deterioration of ventricular function during PCI [2]. The high thrombus burden is an independent predictor of major adverse cardiovascular event (MACE) and infarct-related 
artery stent thrombosis (IRA-ST) in patients treated with drug-eluting stent (DES) for STEMI [3]. However, the mechanisms of plaque thrombus remain unclear and the clinical importance and impact have been underestimated due to limited resolution of intravascular ultrasound (IVUS) and computed tomography [4]. Identifying predictors of the intracoronary thrombus burden may contribute to improved management of intracoronary thrombi in patients with STEMI undergoing pPCI.

Heat shock proteins (HSPs), which are abundant intracellular proteins found in both prokaryotic and eukaryotic organisms, act as chaperones and are involved in protein folding and transport [5]. A considerable amount of experimental evidence indicates that increasing expression of HSPs leads to an important cardio-protective role in ischemia/reperfusion injury, heart failure, cardiac hypertrophy, and arrhythmia, implicating these molecules as potential innovative therapeutic agents [6]. Heat shock protein 27 (Hsp-27) is abundant in cardiac and skeletal muscles and increases in response to stress in order to protect against insults such as ischemia [7-9] Hsp-27 is a $27-\mathrm{kDa}$ chaperone, which can interact with a large number of different proteins and is induced in response to stress [10]. Plasma Hsp-27 concentrations are elevated in the early hours following ACS, but decrease to levels near to those in healthy individuals after about 12 hours from the onset of chest pain [11]. It is unclear whether Hsp-27 levels are associated with thrombus burden and clinical outcomes in patients with STEMI. The aim of this study is to evaluate the association of Hsp-27 levels with thrombus burden and clinical outcomes in patients with STEMI who underwent pPCI.

\section{RESULTS}

The study population consisted of 146 patients with STEMI. The study cohort consisted of 74 males (50.68\%) and 72 females (49.32\%), and the mean age of the participants was $62.8 \pm 10.5$ years. The baseline clinical characteristics between thrombus burden groups are shown in Table 1. Gender, BMI, age, dyslipidemia, DM, smoking, and hypertension were not significantly different between the groups $(P=0.441$, $P=0.501, P=0.617, P=0.558, P=0.122, P=0.120$, and $P=0.421$, respectively). D-Dimer, $\mathrm{CK}-\mathrm{MB}$, hemoglobin, platelet count, TC, TG, LDL-C, HDL-C, and white blood cell count were not significantly different between the groups $(P=0.650, P=0.145, P=0.478, P=0.437, P=0.325$, $P=0.368, P=0.386, P=0.486$, and $P=0.235$, respectively). Previous medications, including $\beta$-blockers, angiotensin converting enzyme inhibitors (ACEI), angiotensin receptor blockers (ARBs), aspirin, nitrates, statins were not significantly different between the groups $(P=0.468, P=0.374, P=0.565$, $P=0.196, P=0.298$, and $P=0.380$, respectively). Culprit vessels were not significantly different between the groups (left anterior descending, circumflex, and right coronary artery; $P=0.768$, $P=0.283$, and $P=0.392$, respectively). The low thrombus burden and high thrombus burden groups did not differ significantly in the Pain-to-ballon time (308 \pm 135 vs. $316 \pm 112 \mathrm{~min}, P>0.05)$ and Door-to-balloon time $(80 \pm 15$ vs. $85 \pm 20 \mathrm{~min}, P>0.05)$ observed in STEMI patients undergoing pPCI. There were no significant differences in the rate of patients treated with stent in low thrombus burden group, compared to that in high thrombus burden group (62 [91.2\%] vs. 74 [94.9\%], $P>0.05)$ as is shown in Table 1. However, patients with low thrombus burden demonstrated significantly higher rates of TIMI grade 3 flow pre-PCI (25 [36.8\%] vs. 11 [14.1\%], $P<0.05)$. Post-PCI coronary flow was slower in patients with pre-PCI high thrombus burden, as demonstrated by lower rates of TIMI grade 3 flow (64 [94.1\%] vs. 65 [83.3\%], $P<0.05)$. As shown in Figure $1 \mathrm{~A}$, patients with high-thrombus burden had higher plasma Hsp-27 levels ([32.0 \pm 8.6 vs. $58.0 \pm 12.3] \mathrm{ng} / \mathrm{mL}$, $P<0.001)$. The plasma levels of C-reactive protein (CRP) in the high thrombus burden group were significantly higher than levels in the low thrombus burden group $([1.312 \pm 0.319$ vs. $2.425 \pm 0.440] \mathrm{mg} / \mathrm{dL}, P<0.05$; Figure 1B). The plasma $\alpha$-tocopherol concentrations in the high thrombus burden group were significantly lower than those in the low thrombus burden group ([53.55 $\pm 6.18 v \mathrm{~s}$. $28.04 \pm 3.27] \mu \mathrm{mol} / \mathrm{L}, P<0.05$; Figure $1 \mathrm{C}$ ). The levels of plasma Hsp-70 were significantly higher in patients with high thrombus burden group than in the low thrombus burden group $([19.16 \pm 3.20$ vs. $31.60 \pm 4.52] \mathrm{ng} / \mathrm{mL}$, $P<0.05$; Figure 1D). Moreover, When statistical analysis was performed in the whole group of combined low thrombus burden and high thrombus burden groups, the plasma levels of Hsp-27 were significantly correlated with the plasma levels of CRP $(r=0.454, P<0.05)$, plasma levels of $\alpha$-tocopherol $(r=0.386, P<0.05)$ and plasma levels of Hsp70 $(r=0.632, P<0.05)$. Using the receiver operating characteristic (ROC) curve analysis, plasma Hsp-27 levels were of significant diagnostic value for high thrombus burden (AUC, 0.847; 95\% CI, 0.775-0.918; $P<0.01$; Figure 2).

The median value of Hsp-27 levels in all patients with STEMI was $45 \mathrm{ng} / \mathrm{mL}$. Univariate and multivariate logistic regression analyses were performed to determine the independent predictors of Hsp-27> $45 \mathrm{ng} / \mathrm{mL}$ (Table 2). The variables univariately associated with $\mathrm{Hsp}-27>45 \mathrm{ng} / \mathrm{mL}$ were D-Dimer (OR 0.865, 95\% CI 0.648-0.963, $P=0.045$ ), CK-MB (OR $0.756,95 \%$ CI $0.401-0.809, P=0.036$ ), smoking (OR 1.645, 95\% CI 1.148-4.128, $P<0.001$ ), WBC (OR 1.135, 95\% CI 1.035-1.428, $P=0.040$ ), high thrombus burden (OR 2.156, 95\% CI 1.636-5.082, $P<0.001$ ), CRP (OR 0.612, 95\% CI 0.358-0.926, $P=0.043$ ), cTnI (OR $3.528,95 \%$ CI 1.286-8.059, $P<0.001$ ), aspirin (OR 0.879, $95 \%$ CI $0.571-0.954, P=0.039)$. The multivariate logistic regression analysis demonstrated that high thrombus burden (OR 3.260, 95\% CI 1.268-6.362, $P<0.001$ ), smoking (OR $1.678,95 \%$ CI 1.012-4.796, $P=0.020$ ), and cTnI (OR $2.829,95 \%$ CI $1.025-6.426, P=0.002$ ) were positively correlated with incidence of Hsp-27> $45 \mathrm{ng} / \mathrm{mL}$. Patients 
Table 1: Baseline clinical characteristics according to thrombus burden ${ }^{\mathrm{a}}$

\begin{tabular}{|c|c|c|c|}
\hline Variable & Low Thrombus Burden $(n=68)$ & High Thrombus Burden $(n=78)$ & $P$ value \\
\hline Male & $36(52.9 \%)$ & $38(48.7 \%)$ & 0.441 \\
\hline $\operatorname{BMI}\left(\mathrm{kg} / \mathrm{m}^{2}\right)$ & $23 \pm 2.2$ & $24 \pm 2.5$ & 0.501 \\
\hline Age (years) & $62.5 \pm 11.3$ & $63.2 \pm 10.2$ & 0.617 \\
\hline Hypertension & $24(35.3 \%)$ & $30(38.5 \%)$ & 0.421 \\
\hline DM & $18(26.5 \%)$ & $19(24.4 \%)$ & 0.122 \\
\hline Dyslipidemia & $30(44.1 \%)$ & $35(44.9 \%)$ & 0.558 \\
\hline Smoking & $30(44.1 \%)$ & $36(46.2 \%)$ & 0.120 \\
\hline $\mathrm{TC}(\mathrm{mg} / \mathrm{dL})$ & $189 \pm 39$ & $172 \pm 65$ & 0.325 \\
\hline TG (mg/dL) & $109 \pm 25$ & $102 \pm 18$ & 0.368 \\
\hline LDL-C (mg/dL) & $118 \pm 32$ & $106 \pm 22$ & 0.386 \\
\hline HDL-C (mg/dL) & $45 \pm 7.2$ & $50 \pm 6.8$ & 0.486 \\
\hline White blood cell count, $\times 10^{9} / \mathrm{L}$ & $10.0 \pm 4.2$ & $11.2 \pm 3.0$ & 0.235 \\
\hline Platelet count, $\times 10^{9} / \mathrm{L}$ & $223 \pm 56$ & $218 \pm 46$ & 0.437 \\
\hline Hemoglobin, g/dL & $14.2 \pm 2.6$ & $14.4 \pm 2.8$ & 0.478 \\
\hline D-Dimer $(\mu \mathrm{g} / \mathrm{L})$ & $128.72 \pm 98.58$ & $112.26 \pm 86.53$ & 0.650 \\
\hline CK-MB (IU/L) & $156 \pm 103$ & $171 \pm 126$ & 0.145 \\
\hline \multicolumn{4}{|l|}{ Medications } \\
\hline$\beta$-blockers & $12(17.6 \%)$ & $13(16.7 \%)$ & 0.468 \\
\hline ACEI & $19(27.9 \%)$ & $21(26.9 \%)$ & 0.374 \\
\hline ARB & $19(27.9 \%)$ & $24(30.7 \%)$ & 0.565 \\
\hline Aspirin & $9(13.2 \%)$ & $12(15.4 \%)$ & 0.196 \\
\hline Nitrates & $16(23.5 \%)$ & $18(23.1 \%)$ & 0.298 \\
\hline Statins & $27(39.7 \%)$ & $36(46.2 \%)$ & 0.380 \\
\hline \multicolumn{4}{|l|}{ Culprit vessel } \\
\hline LAD & $34(50.0 \%)$ & $42(53.8 \%)$ & 0.768 \\
\hline RCA & $22(32.4 \%)$ & $26(33.3 \%)$ & 0.283 \\
\hline LCX & $12(17.6 \%)$ & $10(12.8 \%)$ & 0.392 \\
\hline patients treated with stent & $62(91.2 \%)$ & $74(94.9 \%)$ & 0.156 \\
\hline Door-to-ballon time (min) & $80 \pm 15$ & $85 \pm 20$ & 0.369 \\
\hline Pain-to-ballon time (min) & $308 \pm 135$ & $316 \pm 112$ & 0.278 \\
\hline TIMI grade 3 flow pre-PCI & $25(36.8 \%)$ & $11(14.1 \%)$ & $<0.05$ \\
\hline TIMI grade 3 flow post- PCI & $64(94.1 \%)$ & $65(83.3 \%)$ & $<0.05$ \\
\hline
\end{tabular}

${ }^{a}$ Values are given as means \pm SD or absolute numbers with relative frequencies.

Abbreviations: BMI, body mass index; DM, Diabetes mellitus; TC, total cholesterol; TG, triglyceride; LDL-C, low-density lipoprotein cholesterol; HDL-C, high-density lipoprotein cholesterol; CK-MB, creatine kinase-MB; ACEI, angiotensin converting enzyme inhibitors; ARB, angiotensin receptor blockers; $\mathrm{CCB}$, calcium channel blockers; LAD, left anterior descending artery; LCX, left circumflex artery; RCA, right coronary artery.

with high thrombus burden had a much higher risk of Hsp-27 $>45 \mathrm{ng} / \mathrm{mL}$ than patients with low thrombus burden (Table 2).

At 180-day follow-up, the high thrombus burden group demonstrated a significant trend toward increased incidence of death (4 [5.9\%] vs. 7 [9.0\%], $P<0.05$ ). Moreover, differences were noted between the Hsp-27 $<45 \mathrm{ng} / \mathrm{mL}$ and Hsp-27 $>45 \mathrm{ng} / \mathrm{mL}$ group in death (3 $[5.8 \%]$ vs. $8[8.5 \%], P<0.05)$. Univariate and multivariate cox regression analyses were performed to determine the independent predictors of MACE at 180-day follow-up for patients (Table 3). The univariately associated variables with MACE were Hsp-27 > $45 \mathrm{ng} / \mathrm{mL}$ (HR 2.945, 95\% CI 1.839-6.451, $P<0.001$ ), dyslipidemia (HR 1.780,
95\% CI 1.307-2.280, $P=0.046$ ), hypertension (HR 1.759, 95\% CI 1.314-2.029, $P=0.025$ ), DM (HR 1.212, 95\% CI $1.012-1.968, P=0.045)$, cTnI (HR $1.232,95 \%$ CI $1.105-1.809, P=0.048)$, smoking (HR 1.748, 95\% CI $1.340-3.148, P<0.001$ ), high thrombus burden (HR $2.665,95 \%$ CI $1.540-5.276, P<0.001$ ), aspirin (HR $0.709,95 \%$ CI $0.326-0.854, P=0.026$ ), statins (HR 0.469, $95 \%$ CI $0.203-0.814, P=0.021$ ), ARB (HR $0.356,95 \%$ CI $0.108-0.614, P=0.018$ ) (Table 3). The multivariate cox regression analysis demonstrated that Hsp-27 > $45 \mathrm{ng} / \mathrm{mL}$ (HR 2.801, 95\% CI 1.296-4.789, $P=0.001$ ), high thrombus burden (HR 2.620, 95\% CI 1.240-4.542, $P=0.001$ ), smoking (HR 1.672, 95\% CI 1.471-2.994, 
$P=0.032)$ were positively correlated with incidence of MACE. Patients with Hsp-27> $45 \mathrm{ng} / \mathrm{mL}$ had a much higher risk of MACE than patients with Hsp-27 $<45 \mathrm{ng} / \mathrm{mL}$ (Table 3). Kaplan-Meier survival analysis demonstrated that MACE-free survival at 180-day follow-up was significantly lower in patients with Hsp-27 $>45 \mathrm{ng} / \mathrm{mL}$ $(\log$ rank $=10.28, P<0.001$; Figure $3 \mathrm{~A})$ or with high thrombus burden (log rank $=8.36, P<0.001$; Figure $3 \mathrm{~B})$. Correlation analysis showed that Hsp-27 levels was

A

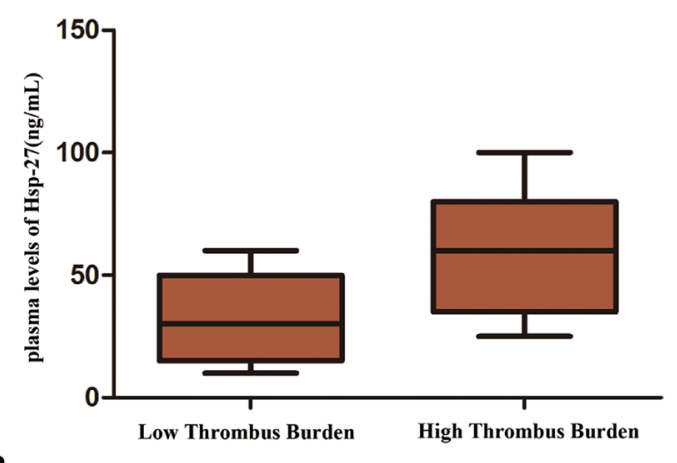

B

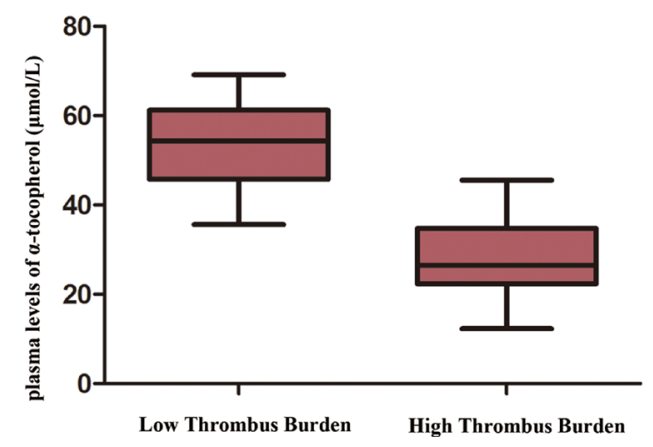

positively correlated with pro-BNP $(r=0.624, P<0.05$; Figure 4A) and negatively correlated with LVEF from echocardiography $(r=-0.528, P<0.05$; Figure 4B).

\section{DISCUSSION}

Hsp-27 constituted essential components of protein synthesis system and function as molecular chaperones to prevent proteins from misfolding and aggregating [12].

C

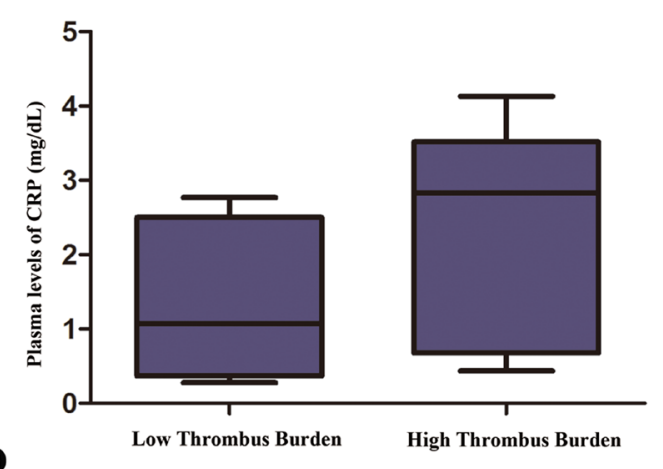

D

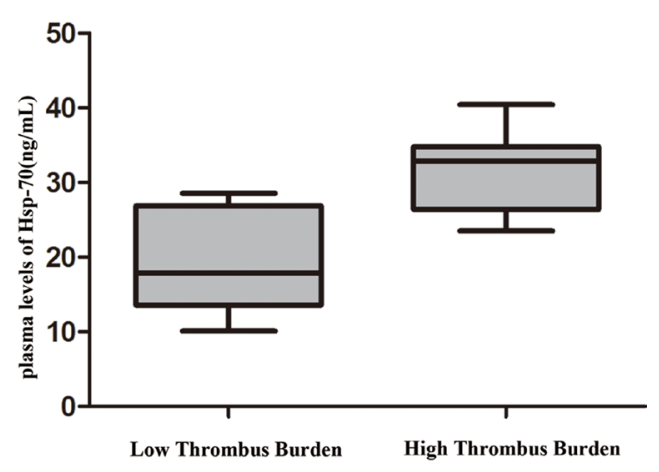

Figure 1: Comparison of plasma heat shock protein 27 (Hsp-27) levels between low and high thrombus burden groups $(P<\mathbf{0 . 0 1})$.

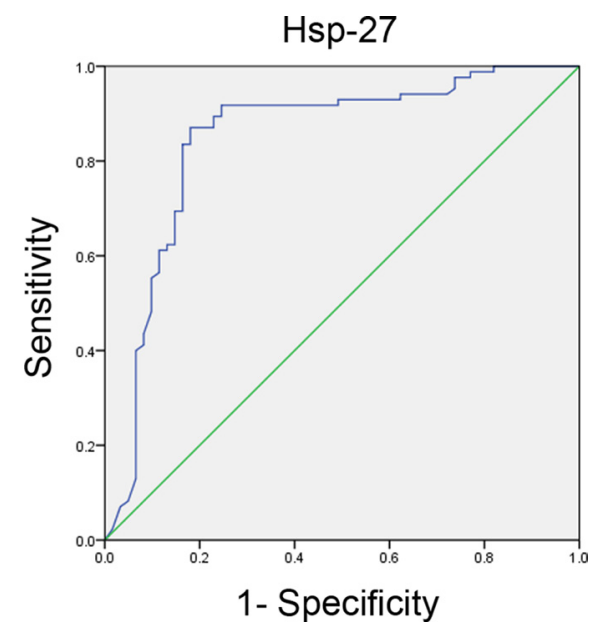

Figure 2: The receiver-operating characteristic (ROC) curve of plasma Hsp-27 for predicting high thrombus burden (AUC, 0.847; 95\% CI, 0.775-0.918; $P<0.01$ ). 
Table 2: Effects of variables on the Hsp-27 $>45 \mathrm{ng} / \mathrm{mL}$ in univariate and multivariate logistic regression analyses

\begin{tabular}{lcc}
\hline Variables & OR $(\mathbf{9 5 \%} \mathbf{C I})$ & $\boldsymbol{P}$ Value \\
\hline Univariate analysis & & \\
D-Dimer & $0.865(0.648-0.963)$ & 0.045 \\
CK-MB & $0.756(0.401-0.809)$ & 0.036 \\
Smoking & $1.645(1.148-4.128)$ & $<0.001$ \\
WBC & $1.135(1.035-1.428)$ & 0.040 \\
High thrombus burden & $2.156(1.636-5.082)$ & $<0.001$ \\
CRP & $0.612(0.358-0.926)$ & 0.043 \\
cTnI & $3.528(1.286-8.059)$ & $<0.001$ \\
Aspirin & $0.879(0.571-0.954)$ & 0.039 \\
Multivariate analysis & & \\
High thrombus burden & $3.260(1.268-6.362)$ & 0.001 \\
Smoking & $1.678(1.012-4.796)$ & 0.020 \\
cTnI & $2.829(1.025-6.426)$ & 0.002 \\
\hline
\end{tabular}

Abbreviations: CI, confidence interval; OR, odds ratio; WBC, White blood cell; CK-MB, creatine kinase MB; CRP, C-reactive protein; cTnI, Cardiac troponin T.

Table 3: Univariate and multivariate cox regression analyses of major adverse cardiovascular events

\begin{tabular}{lcc}
\hline Variables & HR $(\mathbf{9 5} \% \mathbf{C I})$ & $\boldsymbol{P}$ Value \\
\hline Univariate analysis & & $<0.001$ \\
Hsp-27 $>45 \mathrm{ng} / \mathrm{mL}$ & $2.945(1.839-6.451)$ & 0.046 \\
Dyslipidemia & $1.780(1.307-2.280)$ & 0.025 \\
Hypertension & $1.759(1.314-2.029)$ & 0.045 \\
DM & $1.212(1.012-1.968)$ & 0.048 \\
cTnI & $1.232(1.105-1.809)$ & $<0.001$ \\
Smoking & $1.748(1.340-3.148)$ & $<0.001$ \\
High thrombus burden & $2.665(1.540-5.276)$ & 0.026 \\
Aspirin & $0.709(0.326-0.854)$ & 0.021 \\
Statins & $0.469(0.203-0.814)$ & 0.018 \\
ARB & $0.356(0.108-0.614)$ & 0.001 \\
Multivariate analysis & & 0.001 \\
Hsp-27 $>45 \mathrm{ng} / \mathrm{mL}$ & $2.801(1.296-4.789)$ & 0.032 \\
High thrombus burden & $2.620(1.240-4.542)$ & \\
Smoking & $1.672(1.471-2.994)$ & \\
\hline
\end{tabular}

Abbreviations: DM, Diabetes mellitus; cTnI, Cardiac troponin T; ARB, angiotensin receptor blockers.

Moreover, it is now well established that Hsp-27 play important cardioprotective roles in myocardial ischemia reperfusion pathological settings [12]. The response of the post-infarcted heart to ischemia in an experimental model of ischemia and reperfusion injury early after acute myocardial infarction (AMI) was significantly associated with 1.7 fold increase in the expression of the Hsp-27 in AMI hearts vs SHAM [13]. The present results showed that plasma Hsp-27 levels and high thrombus burden were independent predictors of clinical outcomes in patients with STEMI who underwent pPCI. Recognition of molecular chaperone and cardioprotective functions of Hsp-27 is fostering active investigations into the potential of Hsp-27 as therapeutic targets for STEMI undergoing primary PCI. Gaining insight into recent advances may have profound implications in the development of Hsp27-based clinical studies on AMI patients.

High thrombus burden, subsequent distal embolization, and myocardial no-reflow remain a large obstacle that may negate the benefits of urgent coronary revascularization in patients with STEMI. Higher thrombus grade is associated with larger infarct size and slightly worse LV function [14]. High thrombus burden was an independent predictor of mortality (hazard ratio $[\mathrm{HR}] 1.76, P=0.023$ ) and MACE (HR 1.88, $P=$ 0.001 ). Reduced high thrombus burden by glycoprotein $\mathrm{IIb} / \mathrm{III}$ a antagonists or thrombectomy improved in both epicardial and microvascular perfusion, and was 
associated with improved ST-segment resolution by 90 minutes [15]. Intracoronary thrombus management remains an important issue to be resolved. Therefore, in this study, we investigated the relationship between Hsp27 levels and thrombus burden, and whether as a potential novel biomarker for prognosis in patients with STEMI. The present study demonstrated that Hsp-27 > $45 \mathrm{ng} / \mathrm{mL}$ and high thrombus burden, were positively correlated with incidence of MACE by multivariate cox regression analysis. MACE-free survival at 180-day follow-up was significantly lower in patients with Hsp-27> $45 \mathrm{ng} / \mathrm{mL}$ or with high thrombus burden analyzed by Kaplan-Meier survival analysis.

A

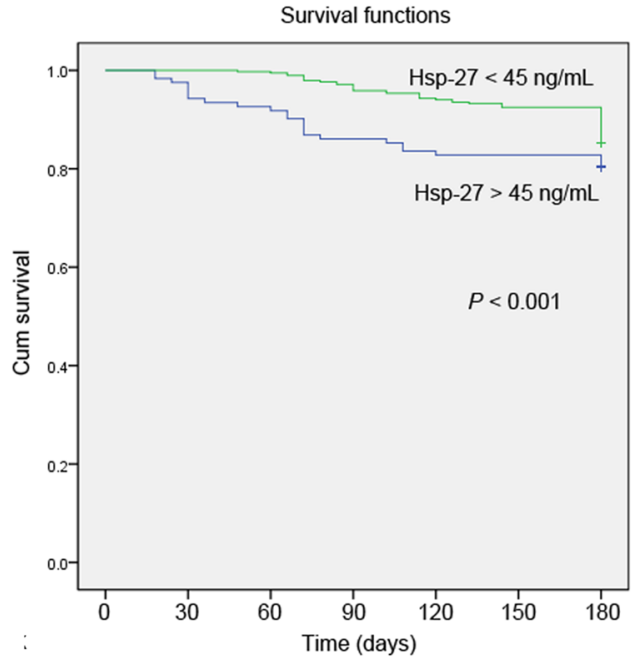

Number at Risk

$\begin{array}{llllllll}\text { Hsp- }-27<45 \mathrm{ng} / \mathrm{mL} & 52 & 52 & 50 & 49 & 47 & 46 & 46\end{array}$

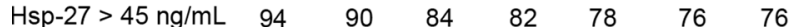

Hsp-27, which belongs to the family of small HSPs that bind denatured proteins following cell stress to prevent aggregation, is an abundant protein selectively expressed in myocardial muscle cells. Hsp-27 renders itself an excellent candidate for cardioprotection against apoptosis, infarction, and oxidative stress during myocardial ischemic injury or hypoxic stress [16]. Previous studies suggested that overexpression of Hsp27 prevents apoptosis by ischemia-reoxygenation in myocardial cells and that overexpression of Hsp-27 in transgenic mice protected the heart against the damaging effects of ischemia/reperfusion [17]. Hsp-27 expression may protect the heart against post-ischemic contractile

B

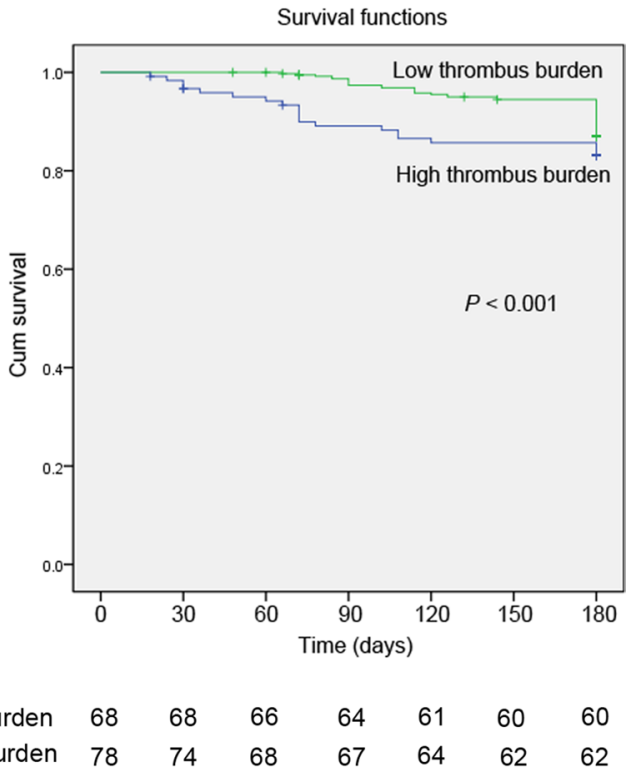

Figure 3: (A) Kaplan-Meier major adverse cardiovascular event (MACE)-free survival curves at 180-day follow-up for patients between Hsp-27 $>45 \mathrm{ng} / \mathrm{mL}$ and Hsp-27 $<45 \mathrm{ng} / \mathrm{mL}$ ( $\log$ rank = 10.28, $P<0.001)$. (B) Kaplan-Meier major adverse cardiovascular event (MACE)free survival curves at 180-day follow-up for patients between high thrombus burden and low thrombus burden $(\log \operatorname{rank}=8.36, P<0.001)$.

A

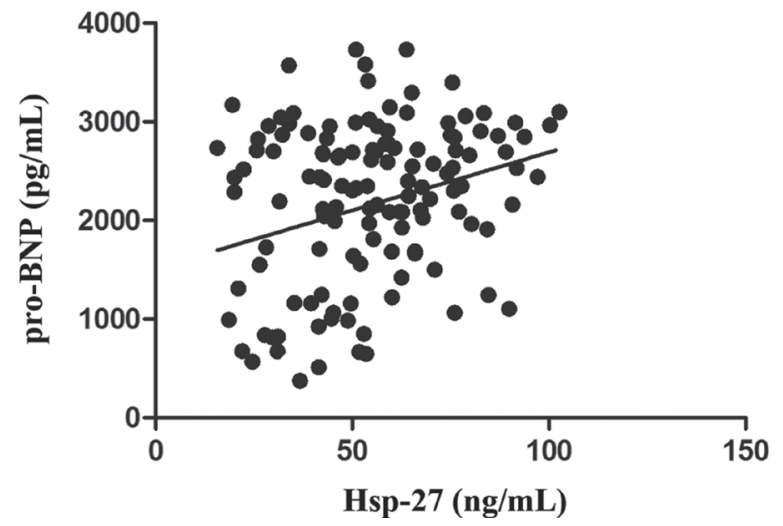

B

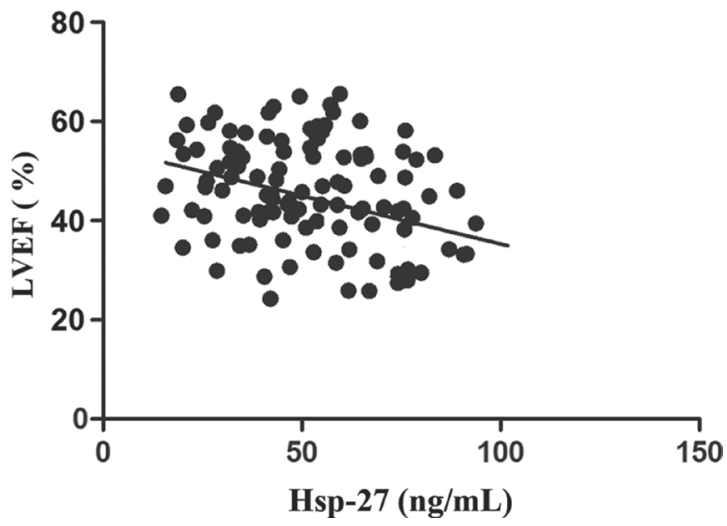

Figure 4: Patterns of circulating plasma Hsp-27 levels in STEMI patients. (A) Correlations between plasma Hsp-27 levels and pro-BNP in STEMI patients $(r=0.624, P<0.05)$. (B) Correlations between plasma Hsp-27 levels and left ventricular ejection fraction (LVEF) in STEMI patients $(r=-0.528, P<0.05)$. STEMI, ST-segment elevation myocardial infarction. 
dysfunction via stabilizing myofilaments, in particular $\mathrm{cTnI}$ and $\mathrm{cTnT}$ [17]. Additionally, investigators observed that patients with dilated cardiomyopathy (DCM) exhibit a two-fold increase in Hsp-27 expression in the heart compared to healthy control patients [18].

MI usually occurs when the fibrous cap overlying an atherosclerotic plaque in a coronary artery ruptures. The resulting exposure of blood to the atherosclerotic material then triggers thrombus formation, which occludes the artery. Plaque accumulation can impair blood flow, causing health complications, escalating to the grave consequence of plaque "rupture," exposing pro-thrombotic material from the plaque to the blood and causing occlusion of the artery [19]. However, the biological function and clinical association of Hsp-27 with thrombus burden in patients with STEMI is not clear. Several lines of evidence support the emerging role of Hsp-27 in modulating atheroprogression, by altering the inflammatory components of atherosclerotic plaques and subsequent thrombus formation [20,21]. Cardiopulmonary bypass had no significant effect on the induction of changes in anti-Hsp-27 levels, which may be due to the formation of immune complexes of antigen-antibody, and antibody levels were higher at the time of discharge [22]. However, serum levels of Hsp-27 are increased in patients undergoing on-pump CABG operation as compared with off-pump CABG technique, owing to spillage of known immune modulatory and apoptosis-associated proteins after CABG operation [23]. Patients with acute cardiac chest pain on admission and approximately $12 \mathrm{~h}$ after the acute event had significantly higher Hsp-27 antibody levels than controls [24]. The present study demonstrated that patients with high-thrombus burden had higher plasma Hsp-27 levels (Figure 1A), and high thrombus burden was an independent predictor of Hsp-27 levels $>45 \mathrm{ng} / \mathrm{mL}$ (Table 2). The plasma Hsp-27 levels were of significant diagnostic value for high thrombus burden by the ROC curve analyses (Figure 2).

Hsp-27 belongs to a family of widespread stress proteins termed small Hsps (sHsps), which is found particularly in several cell types such as cardiomyocytes, endothelial cells, leukocytes and platelets involving in cardiovascular disease [25]. In addition to its chaperoning functions, Hsp-27 also appears to be involved in a diverse range of cellular functions, promoting cell survival through effects on the apoptotic pathway and plays important roles in myocardial infarction and atherosclerosis [25]. Hsp-27 is highly expressed in the heart, and it has been previously studied in myocardial protection models [26, 27]. Hsp27 which has been shown to localize on the sarcomere in cardiomyocytes, comes into circulation from destroyed cardiac cells in the infarct zone of the myocardium during early acute myocardial infarction [28]. Martin et al reported that decreasing the level of endogenous Hsp27 resulted in an enhancement of the damaging effects of a subsequent ischemic stimulus [29, 30]. Ghayour-
Mobarhan et al. have previously reported that Hsp-27 antibody titers were found to be raised in the first $12 \mathrm{~h}$ after the onset of acute myocardial infarction, but decreased to normal in the second $12 \mathrm{~h}$, which was similar to our results in the present study [31]. Plasma Hsp-27 antibody titres are high in patients with chest pain who have suffered an acute myocardial infarction or have unstable angina, being higher in the former, and possibly related to the extent of myocardial ischemia or necrosis [31]. It was also reported that an increased expression of Hsp-27 from infarcted myocardial tissue was released into the peripheral circulation several hours following a stroke [25]. Moreover, Hsp-27, which is also expressed by endothelial cells and may stimulate autoimmune response, has roles in stabilizing atherosclerotic plaque, inhibition of plaque development and inhibition of endothelial cell injury through different mechanisms [32, 33]. Additionally, Hsp-27, also present in platelets, may be more involved in controlling actin polymerization during the platelet shape change and subsequent aggregation, which involving in thrombus formation in STEMI [34]. Hsp-27 may also play roles in inhibition of platelet aggregation, cardioprotection in ischemic events and enhancing post ischemic outcome $[34,35]$.

There is close causal link between high levels of Hsp-27 in patients' plasma and high risks of thrombus burden. Firstly, Plasma Hsp-27 levels are high in patients with STEMI who have suffered the high thrombus burden or low thrombus burden, being higher in the former, and possibly related to the extent of myocardial ischemia or necrosis [31]. Hsp-27 was found to be raised partly due to the high thrombus burden, which caused the more heavy myocardial ischemia or necrosis compared the low thrombus burden group, but not as 'thrombogenic' protein. The higher plasma Hsp-27 levels and high thrombus burden during early STEMI were positively correlated with incidence of MACE at 180-day followup in the present study. Secondly, The fact that the higher expression of Hsp-27 levels was observed in the high thrombus burden group than low thrombus burden is probably because this is where the inflammatory processes and oxidative stress were more active [31]. Compared to the high thrombus burden group, the decrease of Hsp-27 expression in the low thrombus burden may be due to an increase of proteolytic activity in region of the plaque. The proteolytic activity has also been described to play an important role in the thrombotic occlusion of the coronary artery on the grounds of atherosclerotic plaque, which is considered the ultimate step in STEMI [36]. Ventura et al. demonstrated that the lower levels of Hsp-27 expressed by atherosclerotic plaques could be a result of its degradation by proteolysis [21]. There is a significant relationship between Hsp-27 concentrations in plasma and the proteolytic activity [21]. The increase of Hsp-27 expression in high thrombus may be due to a decrease of proteolytic activity in this region of heavy thrombus [37]. 
Patients with high-thrombus burden had higher plasma Hsp-27 levels ([32.0 \pm 8.6 vs. $58.0 \pm 12.3] \mathrm{ng} / \mathrm{mL}$, $P<0.001)$ in the present study. Finally, the increase of Hsp27 releasing from leukocytes and platelets to blood was also related to the complexity of the thrombus formation on atherosclerotic plaques in high thrombus burden [36].

It is interesting and possible to artificially decrease the risk of thrombus burden by targeting the Hsp-27 in patients with STEMI. Firstly, statins (atorvastatin) could decrease the Hsp-27 levels of the proteins secreted by cultured atherosclerotic plaques as well as serum cholesterol [38]. Plasma Hsp-27 concentrations have also been reported to be correlated with total serum cholesterol concentrations in patients with acute coronary syndrome [20]. Interestingly, Hsp-27 detected by PD-Quest analysis as differentially released by atherosclerotic plaques significantly decreased after addition of atorvastatin compared with that in atheroma plaque supernatants in the absence of atorvastatin, in agreement with the proteomic analysis [38]. Statins are also capable of decreasing the levels of different inflammatory proteins in both carotid atherosclerotic plaques and in the blood of patients with carotid atherosclerosis [39]. Secondly, SHIN et al cardiac rehabilitation therapy (CRT) and a combination of CRT with stain treatment (COM) reduced antibody titers to Hsp-60 and Hsp-70 in patients with coronary artery disease (CAD) after percutaneous coronary intervention [40]. There was a significant correlation between antibody titers to Hsp-27 versus Hsp-60 and Hsp-70, involving in autoimmune process of atherosclerosis [40]. Considering the fact that antibody titers to Hsp-27 are associated with the autoimmune process in CAD, CRT and COM may exert greater effects on reduction in plasma Hsp-27 levels in patients with STEMI undergoing PCI than statin treatment only [40]. Based on the findings obtained in the present study and accumulating evidences, down-regulation of the antibody titers to Hsp-27 by cardiac rehabilitation therapy and statins, intervention consisting of cardiac rehabilitation and statin therapy in STEMI patients was found to be of benefit [40]. Finally, use of abciximab was possible to decrease the risk of thrombus burden by targeting the Hsp-27 releasing from platelets of coronary thrombus in patients with STEMI. Abciximab, a platelet glycoprotein (GP) IIb/IIIa inhibitor, in combination with administration of thrombolytics has been shown to reduce angiographically evident thrombus in patients with STEMI [15]. Hsp-27 has important roles in platelet function [34]. There is evidence suggesting a potential role for Hsp-27 in the regulation of transglutaminase activity in stabilizing fibrin-platelet clots. Phosphorylation of Hsp-27 may also contribute to the inhibitory effects of cGK on platelet function [35]. Hsp-27 may be involved in platelet aggregation by modulating actin polymerization [35].

Our study has several limitations, including the small number of patients in a single center in China. Secondly, we could not explain the exact mechanisms triggering change in Hsp-27 between low thrombus burden and high thrombus burden in patients with STEMI undergoing pPCI. Lastly, although we assessed the plasma Hsp-27 levels before PCI, serial measurements of Hsp-27 might be more useful for evaluating changes in inflammatory status, estimating risk during the follow-up period. Therefore, a multicenter trial with a large study population is necessary to validate the clinical importance of Hsp-27 in the future.

In conclusion, the present study results showed that plasma Hsp-27 was positively correlated with high thrombus burden and the incidence of MACE in patients with STEMI who underwent pPCI. Measuring plasma Hsp-27 levels may substantially improve the risk stratification of high thrombus burden and clinical outcomes in STEMI patients.

\section{MATERIALS AND METHODS}

\section{Study population}

Consecutive patients $(n=146)$ having STEMI within 12 hours from onset of symptoms were prospectively enrolled in the study in the Affiliated Yantai Yuhuangding Hospital of Qingdao University, Yantai, Shangdong, P.R. China from June 2014 to January 2016. PCI was performed in all patients and transradial coronary intervention was the preferred operating approach. STEMI was defined as typical chest pain $>30$ minutes duration with ST-segment elevation $>0.1 \mathrm{mV}$ in at least 2 consecutive leads on the electrocardiogram or new-onset left bundle branch block [41]. Important exclusion criteria included previous myocardial infarction (MI), previous use of thrombolytic agents for index MI, cardiogenic shock, cardiomyopathy, previous stroke within the past 6 months, and known bleeding diathesis [41]. Clinical data, including baseline clinical characteristics, angiographic, and laboratory features were recorded for all patients. All patients gave their informed consent, and the study protocol was approved by the Yantai Yuhuangding Hospital's ethics committee.

\section{Laboratory analysis}

Venous blood samples were collected before PCI in all patients. Whole blood was immediately collected into a tube containing ethylene diaminetetraacetate (EDTA); Then, it was centrifuged at $3000 \times \mathrm{g}$ for $15 \mathrm{~min}$ at $4^{\circ} \mathrm{C}$. After separation, plasma was frozen at $-80^{\circ} \mathrm{C}$ until detection for Hsp-27 levels.. Laboratory assessments included total cholesterol (TC), triglycerides (TG), lowdensity lipoprotein cholesterol (LDL-C), high-density lipoprotein cholesterol (HDL-C), D-Dimer, creatine kinase$\mathrm{MB}$ (CK-MB), and cardiac troponin T (cTnT) levels in the emergency department. The CK-MB assessments were repeated every 6 hours to determine peak levels of CK$\mathrm{MB}$, unless clinical events suggested further measurements until discharge. Hemoglobin, white blood cells, mean 
platelet volume, and platelet counts were measured using a Coulter LH 780 Hematology Analyzer (Beckman Coulter Inc, Miami, FL, USA). proBNP plasma levels were measured in each patient on admission by UniCel DXI800 Access Immunoassay System (Beckman Coulter). Plasma Hsp-27 and Hsp-70 levels were determined using sandwich enzyme-linked immunosorbent assay (ELISA) kits (R\&D Systems, Minneapolis, MN, USA) and a microplate spectrophotometer (Multiskan Spectrum; Thermo Scientific, Waltham, MA, USA) [42]. All ELISA tests were performed according to the manufacturer's instructions. Samples were measured in duplicates. The plasma $\alpha$-tocopherol concentration was measured by high performance liquid chromatography (HPLC) with the use of a reverse-phase column (NovaPak C18, 8100-mm Radial-Pak Cartridge, Waters) [43]. C-reactive protein (CRP) was measured by ELISA according to the manufacturer's instructions (R\&D Systems, Minneapolis, MN, USA). Researchers performing the assays and data analyses were blinded to the groups associated with each sample.

\section{PCI procedure}

All patients also received loading with aspirin (300 $\mathrm{mg}$ ) and clopidogrel (300 $\mathrm{mg}$ ) in the emergency department. Left ventricular ejection fraction (LVEF) was measured by 2-dimensional echocardiogram with Simpson methods before primary PCI. A $100 \mathrm{U} / \mathrm{kg}$ intravenous bolus of unfractionated heparin was administered through the arterial access sheaths to all patients before the procedure, and anticoagulation level was adjusted to maintain an activated clotting time of 250 to 300 seconds. Coronary angiography and intervention started within 30 minutes after admission using radial artery approach. Coronary arteries were visualized in left and right oblique planes with cranial and caudal angles at 30 frames/s. Thrombus burden was assessed as previously defined by the thrombolysis in myocardial infarction (TIMI) study group, and quantitative angiography was performed in an angiographic core laboratory by investigators blinded to treatment assignment [15]. In TIMI thrombus grade 0 , no cineangiography characteristics of thrombus are present; in TIMI thrombus grade 1, possible thrombus is present, with angiography characteristics such as reduced contrast density, haziness, irregular lesion contour, or a smooth convex "meniscus" at the site of total occlusion suggestive; in TIMI thrombus grade 2, there is definite thrombus, with greatest dimensions $\leq 1 / 2$ the vessel diameter; in TIMI thrombus grade 3 , there is definite thrombus but with greatest linear dimension .> 1/2 but, $<2$ vessel diameters; in TIMI thrombus grade 4 , there is definite thrombus, with the largest dimension $\geq 2$ vessel diameters; and in TIMI thrombus grade 5, there is total occlusion [15]. The patients were divided into 2 groups with low and high thrombus burden, respectively. The patients were considered to high thrombus burden if TIMI thrombus grades 4 to 5 were present, and the patients with TIMI thrombus grades 1 to 3 were defined as the lower thrombus burden group [44]. In the present study, the study population undergoing primary PCI was stented with drug-eluting stents (DES), Aspirin and clopidogrel were maintained at 100 and $75 \mathrm{mg} / \mathrm{d}$, respectively. Clopidogrel $(75 \mathrm{mg} / \mathrm{d})$ was given for 12 months after DES implantation according to the current guideline [3].

\section{Clinical definitions and follow-up}

The diagnosis of diabetes mellitus (DM) was based on previous history of DM treated with or without drugs. Hypercholesterolemia was diagnosed by total cholesterol concentration $\geq 200 \mathrm{mg} / \mathrm{dL}$, and hypertension was defined as systolic pressure $\geq 140 \mathrm{~mm} \mathrm{Hg}$ and/or a diastolic pressure $\geq 90 \mathrm{~mm} \mathrm{Hg}$ at least 2 times or if the individual was taking antihypertensive medication [45]. Patients were defined smokers if they smoked $\geq 1$ cigarette/d at the time of admission or in the preceding 12 months [45]. Followup was performed through the outpatient department and telephone interviews at 180 days after discharge at the discretion of the attending physician. The primary endpoints were combined MACE (including death, reinfarction, and target vessel revascularization [TVR]), LVEF, lifethreatening arrhythmia (including ventricular fibrillation or sustained ventricular tachycardia), and heart failure at 180day follow-up. All patients have reached 180-day followup, the time period during which dual anti-platelet therapy was recommended in all patients.

\section{Statistical analysis}

Statistical analysis was performed using SPSS 20.0 software (IBM, USA). Continuous data are presented as mean $\pm \mathrm{SD}$. Categorical variables are presented as counts and percentages. Normally distributed data were analyzed using Student $t$ test and for non-normally distributed data nonparametric Mann-Whitney test was used. Categorical variables were compared by chi-square test. Correlations between variables were determined by Pearson tests. Univariate and multivariate logistic regression analyses were used to determine independent predictors of Hsp$27>45 \mathrm{ng} / \mathrm{mL}$. Receiver operating characteristic (ROC) curve analysis and comparison of the derived area under the curve (AUC) were performed to assess the Hsp-27 as a predictor for high thrombus burden. Univariate and multivariate cox regression analyses were performed to determine independent predictors of MACE. Values were expressed as odds ratio (OR) or hazard ratio (HR), with 95\% confidence interval (CI) and $P$ values. Kaplan-Meier survival was calculated to evaluate the MACE-free survival at 180-day follow-up, with the difference analyzed by the log-rank test. Statistical significance was set at $P<0.05$. 


\section{ACKNOWLEDGMENTS AND FUNDING}

This study was supported by the project of the Science and Technology Development Plan of Yantai City (NO.2013WS225).

\section{CONFLICTS OF INTEREST}

The authors confirm that there are no conflicts of interest.

\section{REFERENCES}

1. O'Gara PT, Kushner FG, Ascheim DD, Casey DE Jr, Chung MK, de Lemos JA, Ettinger SM, Fang JC, Fesmire FM, Franklin BA, Granger CB, Krumholz HM, Linderbaum JA, et al, and American College of Emergency Physicians, and Society for Cardiovascular Angiography and Interventions. $2013 \mathrm{ACCF} / \mathrm{AHA}$ guideline for the management of STelevation myocardial infarction: a report of the American College of Cardiology Foundation/American Heart Association Task Force on Practice Guidelines. J Am Coll Cardiol. 2013; 61:e78-140.

2. Yip HK, Chen MC, Chang HW, Hang CL, Hsieh YK, Fang CY, Wu CJ. Angiographic morphologic features of infarct-related arteries and timely reperfusion in acute myocardial infarction: predictors of slow-flow and noreflow phenomenon. Chest. 2002; 122:1322-32.

3. Grines CL, Bonow RO, Casey DE Jr, Gardner TJ, Lockhart PB, Moliterno DJ, O'Gara P, Whitlow P, and American Heart Association, and American College of Cardiology, and Society for Cardiovascular Angiography and Interventions, and American College of Surgeons, and American Dental Association, and American College of Physicians. Prevention of premature discontinuation of dual antiplatelet therapy in patients with coronary artery stents: a science advisory from the American Heart Association, American College of Cardiology, Society for Cardiovascular Angiography and Interventions, American College of Surgeons, and American Dental Association, with representation from the American College of Physicians. J Am Coll Cardiol. 2007; 49:734-39.

4. Kim SW, Hong YJ, Mintz GS, Lee SY, Doh JH, Lim SH, Kang HJ, Rha SW, Kim JS, Lee WS, Oh SJ, Lee S, Hahn JY, et al. Relation of ruptured plaque culprit lesion phenotype and outcomes in patients with ST elevation acute myocardial infarction. Am J Cardiol. 2012; 109:794-99.

5. Willis MS, Patterson C. Hold me tight: role of the heat shock protein family of chaperones in cardiac disease. Circulation. 2010; 122:1740-51.

6. Tarone G, Brancaccio M. Keep your heart in shape: molecular chaperone networks for treating heart disease. Cardiovasc Res. 2014; 102:346-61.
7. Arrigo AP, Simon S, Gibert B, Kretz-Remy C, Nivon M, Czekalla A, Guillet D, Moulin M, Diaz-Latoud C, Vicart P. Hsp27 (HspB1) and alphaB-crystallin (HspB5) as therapeutic targets. FEBS Lett. 2007; 581:3665-74.

8. Lunz W, Capettini LS, Davel AP, Munhoz CD, da Silva JF, Rossoni LV, Lemos VS, Baldo MP, Carneiro-Junior MA, Natali AJ, de Lacerda LH, Mill JG. L-NAME treatment enhances exercise-induced content of myocardial heat shock protein 72 (Hsp72) in rats. Cell Physiol Biochem. 2011; 27:479-86.

9. Katta A, Thulluri S, Manne ND, Addagarla HS, Arvapalli R, Nalabotu SK, Gadde M, Rice KM, Blough ER. Overload induced heat shock proteins (HSPs), MAPK and miRNA (miR-1 and miR133a) response in insulin-resistant skeletal muscle. Cell Physiol Biochem. 2013; 31:219-29.

10. Kabakov AE, Budagova KR, Bryantsev AL, Latchman DS. Heat shock protein 70 or heat shock protein 27 overexpressed in human endothelial cells during posthypoxic reoxygenation can protect from delayed apoptosis. Cell Stress Chaperones. 2003; 8:335-47.

11. Heidari-Bakavoli AR, Sahebkar A, Mobara N, Moohebati M, Tavallaie S, Rahsepar AA, Kazemi A, Alavi MS, Ghayour-Mobarhan M, Ferns GA. Changes in plasma level of heat shock protein 27 after acute coronary syndrome. Angiology. 2012; 63:12-16.

12. Carra S, Alberti S, Arrigo PA, Benesch JL, Benjamin IJ, Boelens W, Bartelt-Kirbach B, Brundel BJ, Buchner J, Bukau B, Carver JA, Ecroyd H, Emanuelsson C, et al. The growing world of small heat shock proteins: from structure to functions. Cell Stress Chaperones. 2017; 22:601-11.

13. Pantos C, Mourouzis I, Dimopoulos A, Markakis K, Panagiotou M, Xinaris C, Tzeis S, Kokkinos AD, Cokkinos DV. Enhanced tolerance of the rat myocardium to ischemia and reperfusion injury early after acute myocardial infarction. Basic Res Cardiol. 2007; 102:327-33.

14. Ahmed TA, Sorgdrager BJ, Cannegieter SC, van der Laarse A, Schalij MJ, Jukema W. Pre-infarction angina predicts thrombus burden in patients admitted for ST-segment elevation myocardial infarction. EuroIntervention. 2012; 7:1396-405.

15. Gibson CM, de Lemos JA, Murphy SA, Marble SJ, McCabe CH, Cannon CP, Antman EM, Braunwald E, and TIMI Study Group. Combination therapy with abciximab reduces angiographically evident thrombus in acute myocardial infarction: a TIMI 14 substudy. Circulation. 2001; 103:2550-54.

16. Hollander JM, Martin JL, Belke DD, Scott BT, Swanson E, Krishnamoorthy V, Dillmann WH. Overexpression of wild-type heat shock protein 27 and a nonphosphorylatable heat shock protein 27 mutant protects against ischemia/ reperfusion injury in a transgenic mouse model. Circulation. 2004; 110:3544-52.

17. Lu XY, Chen L, Cai XL, Yang HT. Overexpression of heat shock protein 27 protects against ischaemia/reperfusion- 
induced cardiac dysfunction via stabilization of troponin I and T. Cardiovasc Res. 2008; 79:500-08.

18. Knowlton AA, Kapadia S, Torre-Amione G, Durand JB, Bies R, Young J, Mann DL. Differential expression of heat shock proteins in normal and failing human hearts. J Mol Cell Cardiol. 1998; 30:811-18.

19. Hansson GK, Libby P. The immune response in atherosclerosis: a double-edged sword. Nat Rev Immunol. 2006; 6:508-19.

20. Park HK, Park EC, Bae SW, Park MY, Kim SW, Yoo HS, Tudev M, Ko YH, Choi YH, Kim S, Kim DI, Kim YW, Lee $\mathrm{BB}$, et al. Expression of heat shock protein 27 in human atherosclerotic plaques and increased plasma level of heat shock protein 27 in patients with acute coronary syndrome. Circulation. 2006; 114:886-93.

21. Martin-Ventura JL, Duran MC, Blanco-Colio LM, Meilhac O, Leclercq A, Michel JB, Jensen ON, Hernandez-Merida S, Tuñón J, Vivanco F, Egido J. Identification by a differential proteomic approach of heat shock protein 27 as a potential marker of atherosclerosis. Circulation. 2004; 110:2216-19.

22. Rahsepar AA, Mirzaee A, Moodi F, Moohebati M, Tavallaie S, Khorashadizadeh F, Eshraghi A, Alavi MS, Zarrabi L, Sajjadian M, Amini M, Khojasteh R, Paydar R, et al. Changes in anti-heat shock protein 27 antibody and C-reactive protein levels following cardiac surgery and their association with cardiac function in patients with cardiovascular disease. Cell Stress Chaperones. 2013; 18:65-74.

23. Szerafin T, Hoetzenecker K, Hacker S, Horvath A, Pollreisz A, Arpád P, Mangold A, Wliszczak T, Dworschak M, Seitelberger R, Wolner E, Ankersmit HJ. Heat shock proteins 27, 60, 70, 90alpha, and 20S proteasome in onpump versus off-pump coronary artery bypass graft patients. Ann Thorac Surg. 2008; 85:80-87.

24. Shams S, Shafi S, Bodman-Smith K, Williams P, Mehta S, Ferns GA. Anti-heat shock protein-27 (Hsp-27) antibody levels in patients with chest pain: association with established cardiovascular risk factors. Clin Chim Acta. 2008; 395:42-46.

25. Ghayour-Mobarhan M, Saber H, Ferns GA. The potential role of heat shock protein 27 in cardiovascular disease. Clin Chim Acta. 2012; 413:15-24.

26. Tallot P, Grongnet JF, David JC. Dual perinatal and developmental expression of the small heat shock proteins [FC12] aB-crystallin and Hsp27 in different tissues of the developing piglet. Biol Neonate. 2003; 83:281-88.

27. Gaitanaki C, Konstantina S, Chrysa S, Beis I. Oxidative stress stimulates multiple MAPK signalling pathways and phosphorylation of the small HSP27 in the perfused amphibian heart. J Exp Biol. 2003; 206:2759-69.

28. Efthymiou CA, Mocanu MM, de Belleroche J, Wells DJ, Latchmann DS, Yellon DM. Heat shock protein 27 protects the heart against myocardial infarction. Basic Res Cardiol. 2004; 99:392-94.
29. Martin JL, Mestril R, Hilal-Dandan R, Brunton LL, Dillmann WH. Small heat shock proteins and protection against ischemic injury in cardiac myocytes. Circulation. 1997; 96:4343-48.

30. Martin JL, Hickey E, Weber LA, Dillmann WH, Mestril $\mathrm{R}$. Influence of phosphorylation and oligomerization on the protective role of the small heat shock protein 27 in rat adult cardiomyocytes. Gene Expr. 1999; 7:349-55.

31. Ghayour-Mobarhan M, Sahebkar A, Parizadeh SM, Moohebati M, Tavallaie S, Rezakazemi-Bajestani SM, Esmaeili HA, Ferns G. Antibody titres to heat shock protein 27 are elevated in patients with acute coronary syndrome. Int J Exp Pathol. 2008; 89:209-15.

32. Pawlowska Z, Baranska P, Jerczynska H, Koziolkiewicz W, Cierniewski CS. Heat shock proteins and other components of cellular machinery for protein synthesis are up-regulated in vascular endothelial cell growth factor-activated human endothelial cells. Proteomics. 2005; 5:1217-27.

33. Lamb DJ, El-Sankary W, Ferns GA. Molecular mimicry in atherosclerosis: a role for heat shock proteins in immunisation. Atherosclerosis. 2003; 167:177-85.

34. Polanowska-Grabowska R, Gear AR. Heat-shock proteins and platelet function. Platelets. 2000; 11:6-22.

35. Butt E, Immler D, Meyer HE, Kotlyarov A, Laass K, Gaestel M. Heat shock protein 27 is a substrate of cGMPdependent protein kinase in intact human platelets: phosphorylation-induced actin polymerization caused by HSP27 mutants. J Biol Chem. 2001; 276:7108-13.

36. Libby P. Molecular and cellular mechanisms of the thrombotic complications of atherosclerosis. J Lipid Res. 2009 (Suppl); 50:S352-57.

37. Wick G. The heat is on: heat-shock proteins and atherosclerosis. Circulation. 2006; 114:870-72.

38. Durán MC, Martín-Ventura JL, Mohammed S, Barderas MG, Blanco-Colio LM, Mas S, Moral V, Ortega L, Tuñón J, Jensen ON, Vivanco F, Egido J. Atorvastatin modulates the profile of proteins released by human atherosclerotic plaques. Eur J Pharmacol. 2007; 562:119-29.

39. Martín-Ventura JL, Blanco-Colio LM, Gómez-Hernández A, Muñoz-García B, Vega M, Serrano J, Ortega L, Hernández G, Tuñón J, Egido J. Intensive treatment with atorvastatin reduces inflammation in mononuclear cells and human atherosclerotic lesions in one month. Stroke. 2005; 36:1796-800.

40. Shin YO, Bae JS, Lee JB, Kim JK, Kim YJ, Kim C, Ahn JK, Bang IK, Lee BK, Othman T, Min YK, Yang HM. Effect of cardiac rehabilitation and statin treatment on anti-HSP antibody titers in patients with coronary artery disease after percutaneous coronary intervention. Int Heart J. 2006; 47:671-82.

41. Steg PG, James SK, Atar D, Badano LP, BlömstromLundqvist C, Borger MA, Di Mario C, Dickstein K, Ducrocq G, Fernandez-Aviles F, Gershlick AH, Giannuzzi P, Halvorsen S, et al, and Task Force on the management 
of ST-segment elevation acute myocardial infarction of the European Society of Cardiology (ESC). ESC Guidelines for the management of acute myocardial infarction in patients presenting with ST-segment elevation. Eur Heart J. 2012; 33:2569-619.

42. Janik S, Schiefer AI, Bekos C, Hacker P, Haider T, Moser J, Klepetko W, Müllauer L, Ankersmit HJ, Moser B. HSP27 and 70 expression in thymic epithelial tumors and benign thymic alterations: diagnostic, prognostic and physiologic implications. Sci Rep. 2016; 6:24267.

43. Göbel Y, Schaffer C, Koletzko B. Simultaneous determination of low plasma concentrations of retinol and tocopherols in preterm infants by a high-performance liquid chromatographic micromethod. J Chromatogr B Biomed Sci Appl. 1997; 688:57-62.
44. Tanboga IH, Topcu S, Aksakal E, Kalkan K, Sevimli S, Acikel M. Determinants of angiographic thrombus burden in patients with ST-segment elevation myocardial infarction. Clin Appl Thromb Hemost. 2014; 20:716-22.

45. Crea F, Gaspardone A, Tomai F, Shoulders C, De Fazio A, Versaci F, Iamele M, Roncaglioni C, Gioffré M, Maseri A, Gioffré PA. Risk factors in schoolchildren associated with a family history of unheralded myocardial infarction or uncomplicated stable angina in male relatives. J Am Coll Cardiol. 1994; 23:1472-78. 